\title{
Electron correlations in two-dimensional small quantum dots
}

\author{
C. Sloggett and O. P. Sushkov \\ School of Physics, University of New South Wales, Sydney 2052, Australia
}

(Dated: November 5, 2018)

\begin{abstract}
We consider circular and elliptic quantum dots with parabolic external confinement, containing $0-22$ electrons and with values of $r_{s}$ in the range $0<r_{s}<3$. We perform restricted and unrestricted Hartree-Fock calculations, and further take into account electron correlations using second-order perturbation theory. We demonstrate that in many cases correlations qualitatively change the spin structure of the ground state from that obtained under Hartree-Fock and spin-density-functional calculations. In some cases the correlation effects destroy Hund's rule. We also demonstrate that the correlations destroy static spin-density waves observed in Hartree-Fock and spin-density-functional calculations.
\end{abstract}

\section{INTRODUCTION}

Recently many theoretical and experimental studies have focused on quantum dots, in which electrons are confined to a mesoscopically small region. These structures are of interest both for studying fundamental physics and for potential nanotechnology applications. With advances in materials science, semiconductor quantum dots have become particularly popular. Most semiconductor dots consist of layers of semiconductor with the electrons confined by the band structure to a layer interface, forming a quasi-2D electron gas. The electrons are further confined in the plane to form the dot, usually by charged gates. Quantum dots containing many electrons are often referred to as "large dots", and those with few (often $N<20$ ) as "small dots". A large dot has a relatively dense electron spectrum and therefore poorly controlled external parameters like the shape of gates, impurities, etc. bring an intrinsic external random component into any description of the dot. For a small dot the uncertainty in the parameters is negligible. The external confining potential can be approximated as $U_{\text {ext }}=\frac{1}{2} m^{*}\left(\omega_{x}^{2} x^{2}+\omega_{y}^{2} y^{2}\right)$, where $m^{*}$ is the effective mass of the electron in the semiconductor, and for circular dots $\omega_{x}=\omega_{y}$. Such small dots are in essence artificial atoms. However, they have the advantage of allowing direct measurement of transport properties, and experimental control over basic parameters such as the size and strength of the confining potential and the number of electrons in the dot. They can also be driven by external magnetic fields that would be inaccessible in atomic physics with realistic magnetic fields.

Small circular quantum dots have been investigated experimentally by Tarucha et $a l^{\frac{1}{n}}$ and by Kouwenhoven et $a l^{2} \stackrel{2}{2}$. These studies have clearly demonstrated the shell structure of the energy spectrum, the effect which one would expect from analogy with atoms or nuclei. Where there is a shell structure we might also expect Hund's rule to be obeyed for the total spin of the dot. Quantum dots have attracted the attention of numerous theoretical studies, for reviews see Refs 3.4 . The electronic structure of $2 \mathrm{D}$ small quantum dots has been studied using spin density functional theory 6.8 .9 .10 .11 .12 as well as by restricted and unrestricted Hartree-Fock methods 13 . These studies have also clearly demonstrated the shell structure of circular dots and they have confirmed the validity of Hund's rule for such dots. As expected, elliptical deformation destroys both the shell structure and Hund's rule. The spin-density-functional calculations also lead to spin-density wave states. However, as has been pointed out by Hirose and Wingreen ${ }^{10}$ such states are artifacts of broken spin symmetry in density-functional theory. This is quite similar to what has been known to occur in unrestricted Hartree-Fock since the work of Overhauser ${ }^{15}$.

As mentioned, there are similarities between small quantum dots and atoms. Therefore theoretical methods used in atomic physics could be efficient in studies of quantum dots. It is known that for few (2-4) electron atoms the best available method is the pair equation method. The configuration-interaction method also works well in this case. However for multi-electron atoms the best available method is Hartree-Fock with further account taken of correlations using many-body perturbation theory. This method is used nowadays in practically all precise calculations for multielectron atoms, see e.g. Refs ${ }^{22.23 .24}$, but to the best of our knowledge it has never been used before for quantum dots. In the present work we perform such calculations. Our results for total energies and shell structure are rather similar to already known ones. However the results concerning the spin structure are different. We never observe a spin density wave in a state with total spin zero. The wave can appear at the Hartree-Fock level, but taking correlations into account restores the rotational symmetry. So, on this we agree with works $\frac{10.15}{15}$ and disagree with ${ }^{6.13}$. Studies have also been done in which the rotational symmetry has been explicitly restored after unrestricted Hartree-Fock via projection techniques $\underline{\underline{14}}$

In the circular dot we have found that the total spin sometimes varies from that predicted by Hund's rule. For $N=10,15,16$, and 17, we found that the ground states have minimal spin as opposed to the previous result of maximal spin within the confines of Hund's rule. We have also studied elliptically deformed small dots, paying special 
attention to the case $\omega_{x} / \omega_{y}=2$. It is well known from nuclear physics ${ }^{16}$ that the shell structure is restored when the ratio of frequencies is a rational number. This is certainly true for a parabolic potential. For a quantum dot the total self-consistent potential is not parabolic (even if the external potential is parabolic), but nevertheless we show that there are some peculiarities in the spectrum of the dot at $\omega_{x} / \omega_{y}=2$.

The structure of the paper is as follows. In Section III we present simple semiclassical estimates which allow us to relate the number of electrons, the strength of the confining potential, and the relative strength of the Coulomb interaction. Section III is devoted to an overview of the computational method. Section IV concerns the two- and six-electron problems in the external parabolic potential. The two-electron problem can easily be solved exactly, while for the six-electron problem we compare to the configuration-interaction study of Reimann et al $\underline{5}$. Comparing these accurate energies with our restricted and unrestricted Hartree-Fock results we demonstrate how unrestricted Hartree-Fock generates the spin density waves and how correlations restore the rotational symmetry. Based on the results of this section it appears that the method gives reliable results to at least $r_{s} \leq 3$, although there appears to be some N-dependence in the accuracy. Finally, Section $\nabla$ presents our results for circular parabolic dots, and Section $\nabla 1$ presents results for elliptical dots.

\section{ANALYTICAL ESTIMATES OF PARAMETERS}

In this section we use atomic units for energy and length

$$
\begin{aligned}
& E_{h}^{*}=\frac{m^{*} e^{4}}{(4 \pi \epsilon \hbar)^{2}} \approx 11 \mathrm{meV}, \\
& a_{B}^{*}=\frac{4 \pi \epsilon \hbar^{2}}{m^{*} e^{2}} \approx 102 \AA \approx 10 \mathrm{~nm} .
\end{aligned}
$$

Here $e$ is the elementary charge, $m^{*}$ is the electron effective mass, and $\epsilon=\kappa \epsilon_{0}$ is the dielectric constant of the material. Numerical values are presented for $m^{*}=0.067 m_{e}$ and $\epsilon=12.9 \epsilon_{0}$, which correspond to the GaAs commonly used in quantum dots. The confining external potential is of the form

$$
U_{e x t}=\frac{1}{2}\left(\omega_{x}^{2} x^{2}+\omega_{y}^{2} y^{2}\right) \rightarrow \frac{1}{2} \omega^{2} r^{2}
$$

Assuming that the number of electrons in the dot is large, $N \gg 1$, we can use the standard semiclassical expression for number density in a $2 \mathrm{D}$ circular dot of radius $R^{17}$

$$
n(r)=n_{0} \sqrt{1-r^{2} / R^{2}}
$$

where $n_{0}=3 N /\left(2 \pi R^{2}\right)$. The electrostatic potential energy at the origin and at the edge of the dot is

$$
\begin{aligned}
U(0) & =\frac{\pi^{2}}{2} n_{0} R, \\
U(R) & =\frac{\pi^{2}}{4} n_{0} R .
\end{aligned}
$$

In the Thomas-Fermi approximation the number density at $r=0$ is related to the Fermi momentum at this point

$$
p_{F}^{2}=2 \pi n_{0}
$$

and the self-consistency equation reads

$$
\epsilon_{F}=\frac{p_{F}^{2}}{2}=\left.\left(U_{e x t}+U\right)\right|_{r=R}-\left.\left(U_{e x t}+U\right)\right|_{r=0} .
$$

At $N \gg 1$ the Fermi energy is small $\left(\propto N^{-1 / 3}\right)$ compared to the self-consistent potential. So Eq. (6) implies that $\left.\left(U_{\text {ext }}+U\right)\right|_{r=R}=\left.\left(U_{\text {ext }}+U\right)\right|_{r=0}$. This gives the standard semiclassical relation between the size of the dot and the number of electrons (see for example Ref ${ }^{18}$ )

$$
\begin{aligned}
R & =\left(\frac{3 \pi N}{4 \omega^{2}}\right)^{\frac{1}{3}} \\
n_{0} & =\left(\frac{6}{\pi^{5}}\right)^{\frac{1}{3}} \omega^{\frac{4}{3}} N^{\frac{1}{3}}
\end{aligned}
$$


The number density of electrons varies from point to point. Therefore the interaction is less important at the centre and more important towards the edge of the dot. To characterize the interaction we define an average $r_{s}$ according to the equation

$$
\pi r_{s}^{2} N=\pi R^{2}, \quad \text { or } \quad R=r_{s} \sqrt{N}
$$

Then, using (7) one finds

$$
r_{s}=\left(\frac{3 \pi}{4}\right)^{\frac{1}{3}} \omega^{-\frac{2}{3}} N^{-\frac{1}{6}}
$$

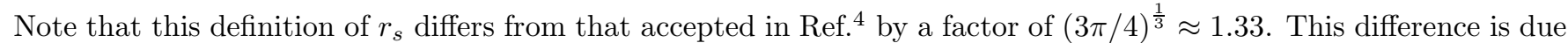
to the semiclassical estimate we use for the dot density in Eq. (3), as opposed to the assumption 4 that the density is roughly flat.

The total energy of the dot scales as

$$
E \propto N^{\frac{3}{2}} r_{s}^{-1}
$$

We will use Eqs. (7), (8), (9), and (10) not only for circular dots, but for elliptic dots as well. In this case we will take $\omega=\sqrt{\omega_{x} \omega_{y}}, R=\sqrt{a b}$.

We stress once more that Eqs. (7), (8), (9), and (10) are written in dimensionless atomic units. Using Eq. (11) one can easily convert them to other units. For example a GaAs quantum dot with $r_{s}=1$ and containing about 20 electrons should have a radius of the order of $R \approx 500 \AA \approx 0.1 \mu \mathrm{m}$. The confining potential $\hbar \omega$ must be on the order of $8 \mathrm{meV}$ once any screening effects from the surrounding layers are considered.

\section{METHOD}

\section{A. The model}

The electron wavefunctions are modeled on a square lattice. Essentially we consider an Anderson model with Hamiltonian

$$
H=\sum_{i \sigma}\left(4+\frac{1}{4}\left(\bar{\omega}_{x}^{2} x_{i}^{2}+\bar{\omega}_{y}^{2} y_{i}^{2}\right)\right) c_{i \sigma}^{\dagger} c_{i \sigma}-\sum_{\langle i j\rangle \sigma} c_{i \sigma}^{\dagger} c_{j \sigma}+\frac{1}{2} \sum_{i j \alpha \beta} \frac{q^{2}}{\left|\mathbf{r}_{i}-\mathbf{r}_{j}\right|} c_{i \alpha}^{\dagger} c_{i \alpha} c_{i \beta}^{\dagger} c_{i \beta}
$$

Here $c_{i \sigma}^{\dagger}$ is the creation operator of an electron with spin projection $\sigma$ ( $\uparrow$ or $\downarrow$ ) at site $i$ of the two-dimensional square lattice with lattice spacing equal to one. $\langle i j\rangle$ represents a sum over nearest neighbor sites. The single particle dispersion corresponding to the Hamiltonian (11) is

$$
\epsilon_{p}=4-2 \cos p_{x}-2 \cos p_{y} \approx p^{2}
$$

At larger momenta the dispersion deviates from quadratic dispersion, so to simulate the real quadratic dispersion we need to have many lattice points within one period of the electron wave function, which is on the order of one atomic unit. For this reason, the units of length used in the Anderson Hamiltonian (11) are not atomic units. The Hamiltonian is written in units in which the lattice spacing is one, where the lattice spacing needs to be significantly smaller than one atomic unit.

The Coulomb interaction $q^{2} /\left|\mathbf{r}_{i}-\mathbf{r}_{j}\right|$ is singular at $i=j$. This is an unphysical singularity as, firstly, if the integral were performed in continuous instead of discrete $2 \mathrm{D}$ space there would be no divergence, and secondly as in a real dot there is transverse (orthogonal to the plane) confinement with some finite effective length, typically on the order of $10 \mathrm{~nm}$.

Here, we avoid adding a finite width to our model in order to be able to compare our results to exact and configuration-interaction $2 \mathrm{D}$ calculations. Instead we consider that when $\mathbf{r}_{i}=\mathbf{r}_{j}$, the effective distance between electrons should not be taken to be zero, but some finite value characteristic of the size of the lattice cell. We obtain an approximate value for the integral at $\mathbf{r}_{i}=\mathbf{r}_{j}$ by treating the wavefunctions as constant over the unit cell and taking the integral of $q^{2} /\left|\mathbf{r}_{i}-\mathbf{r}_{j}\right|$ over the cell. That is, when $\mathbf{r}_{i}=\mathbf{r}_{j}$, we take

$$
\frac{1}{\left|\mathbf{r}_{i}-\mathbf{r}_{j}\right|}=\int_{\text {lattice cell }} \frac{1}{\left|\mathbf{r}-\mathbf{r}^{\prime}\right|} d \mathbf{r} d \mathbf{r}^{\prime} \approx 0.34
$$


where the "inverse characteristic distance" of 0.34 is in units determined by the lattice spacing. The Hartree-Fock results are slightly sensitive to the value given here. However, when correlations are taken into account the results are not sensitive to this value.

As a first approximation for the ground state of the Hamiltonian (11) we use the unrestricted and restricted HartreeFock (HF) methods, modified according to the Optimal Damping Algorithm of Cances and Le Bris 19 . Recall that the $\mathrm{HF}$ equations are of the form

$$
H_{\sigma_{i}} \psi_{i}=\epsilon_{i} \psi_{i}
$$

where $\psi_{i}(r)$ is a single electron orbital which has energy $\epsilon_{i}$, and spin projection on the z-axis $\sigma_{i}= \pm \frac{1}{2}$. The HF Hamiltonian matrix is of the form

$$
\left\langle r\left|H_{\sigma_{i}}\right| r^{\prime}\right\rangle=\left\langle r\left|H_{s}\right| r^{\prime}\right\rangle+\left\langle r\left|U_{\text {dir }}\right| r^{\prime}\right\rangle-\left\langle r\left|U_{\text {exch, } \sigma_{i}}\right| r^{\prime}\right\rangle
$$

where

$$
\begin{aligned}
& \left\langle r\left|U_{d i r}\right| r^{\prime}\right\rangle=\delta_{r r^{\prime}} q^{2} \sum_{\epsilon_{k} \leq \epsilon_{F}} \sum_{r^{\prime \prime}} \frac{\psi_{k}^{\dagger}\left(\mathbf{r}^{\prime \prime}\right) \psi_{k}\left(\mathbf{r}^{\prime \prime}\right)}{\left|\mathbf{r}-\mathbf{r}^{\prime \prime}\right|} \\
& \left\langle r\left|U_{\text {exch }, \sigma_{i}}\right| r^{\prime}\right\rangle=q^{2} \sum_{\epsilon_{k} \leq \epsilon_{F}} \delta_{\sigma_{i} \sigma_{k}} \frac{\psi_{k}^{\dagger}\left(\mathbf{r}^{\prime}\right) \psi_{k}(\mathbf{r})}{\left|\mathbf{r}-\mathbf{r}^{\prime}\right|}
\end{aligned}
$$

are direct and exchange interactions. The single-particle Hamiltonian has only diagonal and nearest neighbour nonzero matrix elements,

$$
\left.\left\langle r\left|H_{s}\right| r^{\prime}\right\rangle=\delta_{r r^{\prime}}\left(4+\frac{1}{4}\left(\bar{\omega}_{x}^{2} x^{2}+\bar{\omega}_{y}^{2} y^{2}\right)\right)-\delta_{<r r^{\prime}}\right\rangle
$$

Hear $\delta_{r r^{\prime}}$ is the usual Kronecker delta. $\delta_{\left\langle r r^{\prime}\right\rangle}=1$ if $r$ and $r^{\prime}$ are nearest neighbours and $\delta_{<r r^{\prime}>}=0$ otherwise. The total energy of the system is

$$
E_{\mathrm{HF}}=\sum_{\epsilon_{i} \leq \epsilon_{F}}\left(\left\langle H_{s}\right\rangle_{i}+\frac{1}{2}\left\langle U_{d i r}\right\rangle_{i}-\frac{1}{2}\left\langle U_{\text {exch }}\right\rangle_{i}\right)=\sum_{\epsilon_{i} \leq \epsilon_{F}}\left(\epsilon_{i}-\frac{1}{2}\left\langle U_{d i r}\right\rangle_{i}+\frac{1}{2}\left\langle U_{\text {exch }}\right\rangle_{i}\right)
$$

In the unrestricted HF (UHF) method the single particle orbitals with spin up are completely independent of those with spin down. Therefore, generally UHF spontaneously violates the rotational invariance of the Hamiltonian (11). The total spin $S$ does not commute with the UHF Hamiltonian and hence only the z-projection of the total spin, $S_{z}$, is conserved. To characterise this effect we introduce the parameter "spin separation"

$$
\Delta n=\frac{1}{2} \int\left|n_{\uparrow}(\mathbf{r})-n_{\downarrow}(\mathbf{r})\right| d \mathbf{r} .
$$

Here $n_{\uparrow}$ is the total density of electrons with spin up and $n_{\downarrow}$ is the total density of electrons with spin down. The spin separation measures the spatial separation of up and down electrons. It can be nonzero even for a state with $S_{z}=0$. Strictly speaking the spontaneous violation of rotational symmetry and hence nonzero value of $\Delta n$ for a state with $S_{z}=0$ is not a physical effect, but a byproduct of the method. Nevertheless, a calculation of $\Delta n$ can shed light on the physics of the system. For example, zero $\Delta n$ indicates that there is no spontaneous symmetry violation. If $\Delta n \approx 1$ in the sector with $S_{z}=0$ there are two possibilities: either the total spin is $S=1$ and we effectively see the state $\left|S=1, S_{z}=0\right\rangle$, or the state is $\left|S=0, S_{z}=0\right\rangle$. By performing the UHF calculation in the sector with $S_{z}=1$ one can try to distinguish between these possibilities.

In the restricted HF (RHF) method the HF equations are exactly the same, but in addition we impose the constraint that electron orbitals with spin up and spin down are identical. Hence RHF automatically respects the spin rotational invariance and describes the state with $S=0$. The RHF total energy is always higher than the UHF total energy.

\section{B. Correlation corrections}

The leading correlation correction to the total energy is given by the diagrams in Fig. 1] The corresponding formula reads 

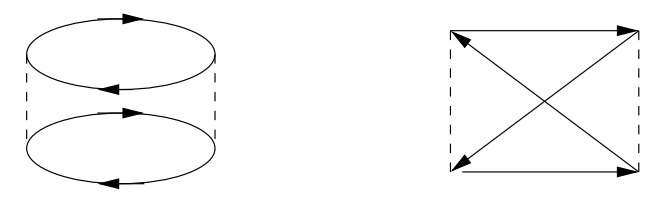

FIG. 1: The leading correlation correction to total energy

$$
\delta E_{\mathrm{corr}}=\frac{1}{2} \sum_{\epsilon_{i}, \epsilon_{j} \leq \epsilon_{F}} \sum_{\epsilon_{m}, \epsilon_{n}>\epsilon_{F}} \frac{|\langle m, n|V| i, j\rangle|^{2}-\langle i, j|V| m, n\rangle\langle m, n|V| j, i\rangle}{\epsilon_{i}+\epsilon_{j}-\epsilon_{m}-\epsilon_{n}}
$$

where the Coulomb matrix element is defined as

$$
\langle m, n|V| i, j\rangle=\delta_{\sigma_{m} \sigma_{i}} \delta_{\sigma_{n} \sigma_{j}} \sum_{\mathbf{r}, \mathbf{r}^{\prime}} \psi_{m}(\mathbf{r}) \psi_{n}\left(\mathbf{r}^{\prime}\right) \frac{q^{2}}{\left|\mathbf{r}-\mathbf{r}^{\prime}\right|} \psi_{i}(\mathbf{r}) \psi_{j}\left(\mathbf{r}^{\prime}\right)
$$

It is well known that in a 3D uniform electron gas the direct diagram in Fig. 1 is logarithmically divergent due to the long-range nature of the Coulomb interaction. Hence it requires consideration of screening effects (higher orders

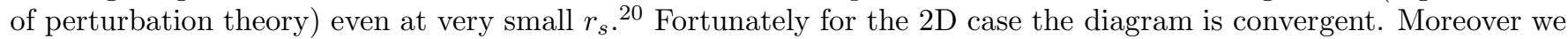
consider a relatively small dot and in this case the second order correlation diagram is convergent even in the $3 \mathrm{D}$ case.

\section{Restricted HF with an odd number of electrons}

There is no difference between an even and an odd number of electrons when performing UHF calculations. However, a restricted Hartree-Fock calculation with an odd number of electrons is not straightforward as the spin-up and spin-down electrons must feel a different exchange potential, breaking the symmetry required by RHF. The Slater determinant of the problem consists of $(N-1) / 2$ doubly occupied orbitals which represent a core with total spin zero, and also one upper orbital which contains only one electron. To preserve the rotational invariance of the core we use the same method used in atomic physics; we average over polarizations of the unpaired electron. This means that we introduce occupation numbers $n_{i}$ where $n_{i}=1$ for any core orbital, $n_{e \uparrow}=n_{e \downarrow}=\frac{1}{2}$ where $e$ is the external orbital, and $n_{i}=0$ otherwise. The Hartree-Fock equations (14)-(18) are modified appropriately. For example instead of Eq. (18), the total energy reads

$$
E_{\mathrm{RHF}}=\sum_{i}\left(n_{i} \epsilon_{i}-\frac{1}{2} n_{i}\left\langle U_{d i r}\right\rangle_{i}+\frac{1}{2} n_{i}\left\langle U_{e x c h}\right\rangle_{i}\right)
$$

This procedure defines our first approximation and it corresponds to the many-body state with total spin $S=\frac{1}{2}$.

The next step is to take into account the fact that the real state contains one $|e \uparrow\rangle$ electron and no $|e \downarrow\rangle$ electron. We use perturbation theory, which automatically guarantees that the total spin remains $S=\frac{1}{2}$. The transition from two external "half-electrons" to one "whole" external electron results in a perturbation acting on the external electron $\Delta V_{e \uparrow}=-\frac{1}{2} \operatorname{Dir}(e)$, and perturbations acting on the core electrons $\Delta V_{i \uparrow}=-\frac{1}{2} \operatorname{Ex}(e)$ and $\Delta V_{i \downarrow}=\frac{1}{2} \operatorname{Ex}(e)$. Here $\operatorname{Dir}(e)$ and $\operatorname{Ex}(e)$ represent direct and exchange interaction with the "external" orbital $\psi_{e}$. Applying these perturbations one finds the following first- and second-order corrections to total energy (22).

$$
\begin{aligned}
\delta E^{(1)} & =-\frac{1}{4}\langle e, e|V| e, e\rangle, \\
\delta E^{(2)} & =\frac{1}{4} \sum_{k>e} \frac{|\langle e, e|V| k, e\rangle|^{2}}{\epsilon_{k}-\epsilon_{e}}+\frac{1}{4} \sum_{i<e} \sum_{k>e} \frac{|\langle i, e|V| e, k\rangle|^{2}}{\epsilon_{k}-\epsilon_{i}}+\frac{1}{4} \sum_{i<e} \sum_{k \geq e} \frac{|\langle i, e|V| e, k\rangle|^{2}}{\epsilon_{k}-\epsilon_{i}} .
\end{aligned}
$$

We use the notation defined in Eq. (21). Note that (23) is not the same as the correlation correction. The correlation energy has to be calculated separately using (20). In (23) we go to second order in order to retain formal accuracy comparable to the correlation terms (20). However, in practice we have found that $\delta E^{(2)}$ is always very small and can be neglected. 

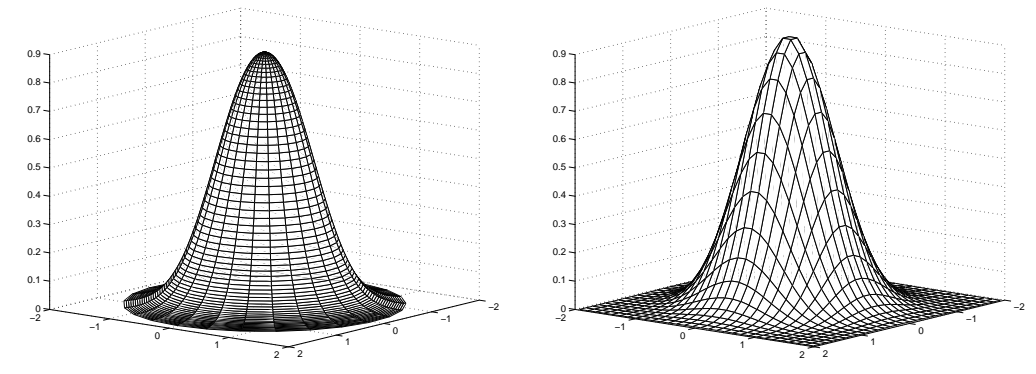

FIG. 2: Exact (left) and Hartree-Fock (right) charge densities (atomic units) for a two-electron dot at $r_{s}=0.77$. In this case restricted and unrestricted Hartree-Fock results are practically identical. Note that $r_{s}$ here is given by the semiclassical estimate made in Section II and, for only two electrons, will not be exactly equal to $E_{\text {Coulomb }} / E_{\text {Kinetic }}$.
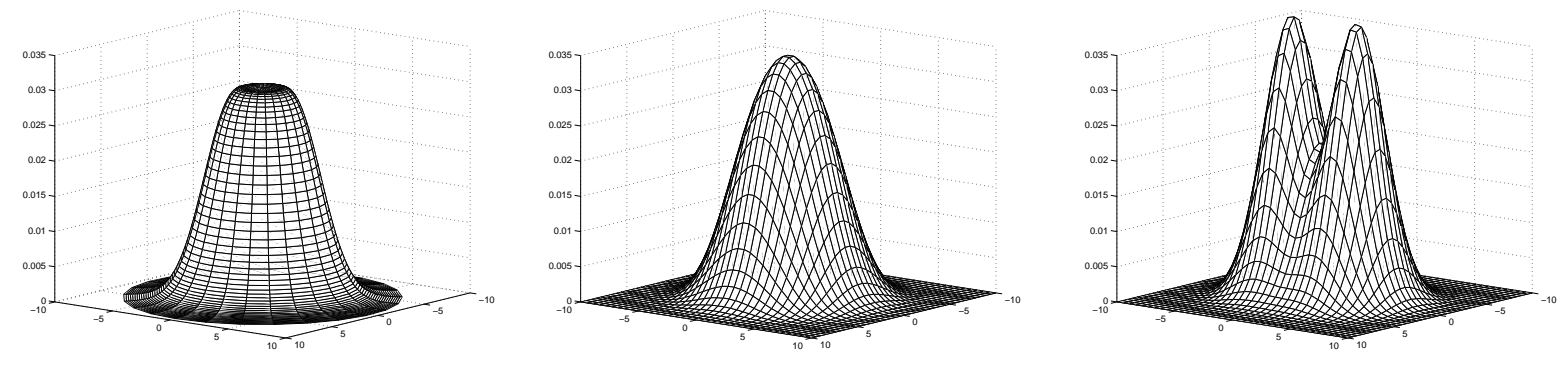

FIG. 3: Exact (left), restricted Hartree-Fock (central), and unrestricted Hartree-Fock (right) charge densities (atomic units) for a two-electron dot at $r_{s}=4.87$. Note that $r_{s}$ here is given by the semiclassical estimate made in Section III and, for only two electrons, will not be exactly equal to $E_{\text {Coulomb }} / E_{\text {Kinetic }}$.

\section{TWO PARTICLES IN A CIRCULAR PARABOLIC POTENTIAL}

The two-body problem can be solved exactly. The Hamiltonian of the problem is

$$
H=\frac{\mathbf{p}_{\mathbf{1}}^{2}}{2 m}+\frac{\mathbf{p}_{\mathbf{2}}^{2}}{2 m}+\frac{1}{2} m \omega^{2}\left(\left|\mathbf{r}_{\mathbf{1}}\right|^{2}+\left|\mathbf{r}_{\mathbf{2}}\right|^{2}\right)+\frac{e^{2}}{\left|\mathbf{r}_{\mathbf{1}}-\mathbf{r}_{\mathbf{2}}\right|}
$$

Using a centre-of-mass coordinate and a relative position coordinate:

$$
\begin{aligned}
\mathbf{R} & =\frac{1}{2}\left(\mathbf{r}_{1}+\mathbf{r}_{2}\right) \\
\mathbf{r} & =\mathbf{r}_{1}-\mathbf{r}_{\mathbf{2}}
\end{aligned}
$$

one can transform the Hamiltonian to

$$
H=-\frac{1}{4 m} \frac{\partial^{2}}{\partial \mathbf{R}^{2}}+m \omega^{2} \mathbf{R}^{2}-\frac{1}{m} \frac{\partial^{2}}{\partial \mathbf{r}^{2}}+\frac{1}{4} m \omega^{2}|\mathbf{r}|^{2}+\frac{e^{2}}{|\mathbf{r}|}
$$

Variables in the corresponding Schroedinger equation are separated and hence one can easily find the exact solution of the problem by solving the radial equation for $\mathbf{r}$ numerically. The ground state has relative orbital angular momentum equal to zero, $l=0$. Therefore, because of Fermi statistics, the spin of the ground state is also zero, $S=0$. The first excitation has $l=S=1$. For clarity, in Fig. [4 we plot values of $E r_{s}$ versus $r_{s}$, where $E$ is the energy in atomic units and $r_{s}$ is defined according to (9) at $N=2$ (see comment ${ }^{21}$ ).

Fig. 惝 shows exact energies for $S=0$ and $S=1$, UHF energies for $S_{z}=0$ and $S_{z}=1$, and the RHF $S=0$ energy. Fig. [b shows the same energies but with second order correlation corrections included (20). In this case the dashed lines show $E_{\mathrm{UHF}}+\delta E_{\mathrm{UHF}}$ at $S_{z}=0$ and $S_{z}=1$.

In Fig. 2] and Fig. 3 we show exact, RHF, and UHF electron densities in the ground state for $r_{s}=0.77$ and $r_{s}=4.87$ respectively. At small $r_{s}$ both the restricted and unrestricted $\left(S_{z}=0\right)$ Hartree-Fock methods give results very close to the exact $S=0$ solution. However, at larger $r_{s}$ the ground state UHF energy is lower than that obtained 

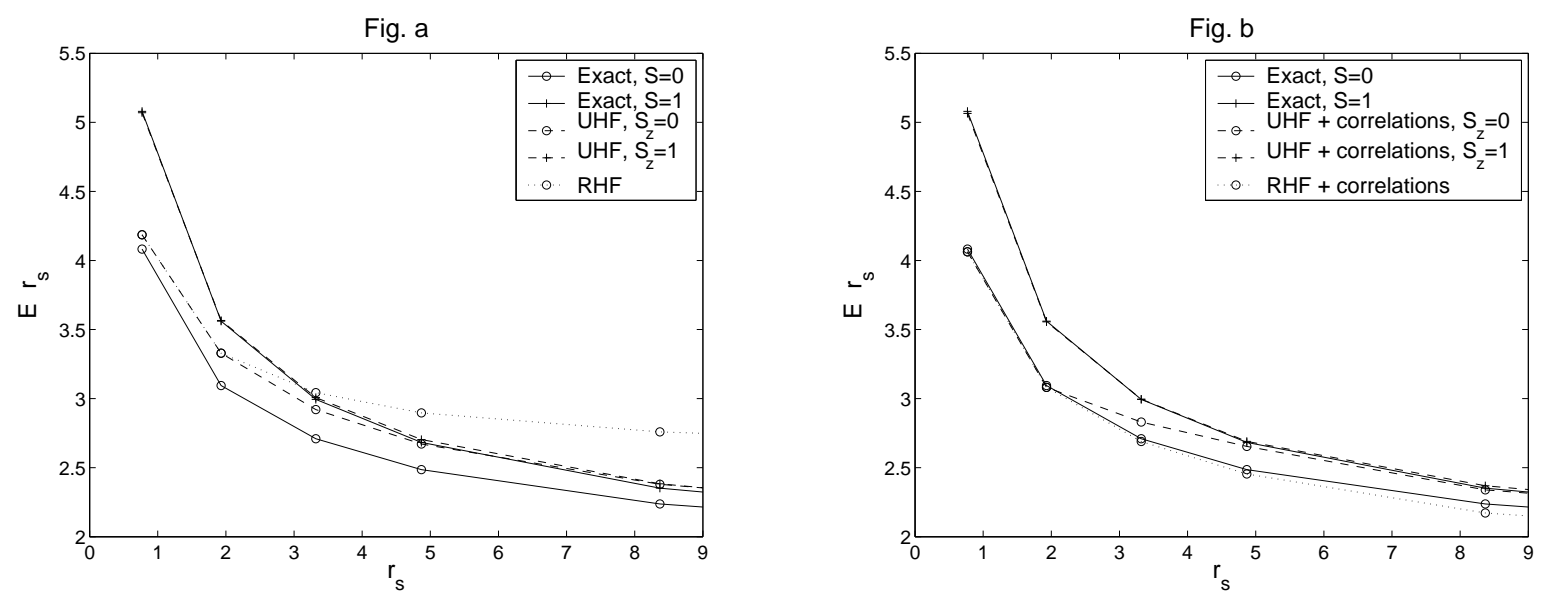

FIG. 4: Total energy versus $r_{s}$ for the two particle problem. We plot the total energy in atomic units multiplied by $r_{s}$. Exact solutions for $S=0$ and $S=1$ are shown in both Fig.a and Fig.b by solid lines. Fig.a also shows the UHF energy with $S_{z}=0$ and $S_{z}=1$ (the dashed lines) and the RHF energy (the dotted line). Fig.b shows the same energies with second-order correlation terms (20) included in all cases. Lines joining data points are given as a guide to the eye only.

in the restricted Hartree-Fock method (see Fig. 四). The UHF energy for the ground state approaches the energy of the first excitation with $S=1$ at larger values of $r_{s}$.

This illustrates that the UHF method spontaneously violates the spin and rotational symmetry of the problem and mixes the true ground state, which has $S=0$, with the state $\left|S=1, S_{z}=0\right\rangle$. At $r_{s}>2$ the $S=1$ component dominates. This leads to early "Wigner crystallization" which is a byproduct of the UHF method. This is confirmed by the pictures of electron density at $r_{s}=4.87$ shown in Fig. 3 There are two separate peaks in the UHF electron density; one corresponds to the electron with spin up and another to the electron with spin down. The UHF spin separation parameter (19) is $\Delta n=1.7$. At the same time neither the exact solution nor the RHF solution indicate any spin separation.

Taking correlations into account drastically changes the situation. The total RHF+correlation energy shown by the dotted line Fig. 40 is close to the exact energy up to very high $r_{s}$ for the two-electron problem and is below the corresponding energy obtained with the UHF+correlations method. Thus the inclusion of correlations leads to the choice of the RHF solution as the ground state, hence restoring the rotational invariance at $S=0$. Certainly at very large $r_{s}$, all Hartree-Fock methods fail.

Fig. [5] gives a similar comparison for the six-electron dot, with configuration-interaction values taken from the

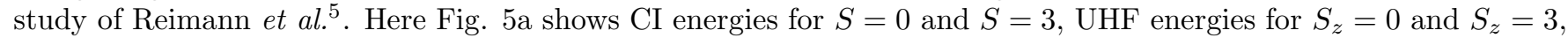
and the RHF $S=0$ energy, while Fig. 4b shows the same energies but with second order correlation corrections included (20). We see the same behaviour as in the two-electron problem, although the third and higher order terms not included in our method are larger when $\mathrm{N}=6$. Again the true ground state is $S=0$, and as $r_{s}$ increases the UHF $S_{z}=0$ solution breaks the symmetry of the problem and converges to the polarised solution. By using RHF as a base for the perturbation theory we remove the spin density waves and restore the rotational invariance of the ground state.

We point out that the UHF and the UHF+correlations method both work pretty well for the polarised $S=1$ and $S=3$ states, see Fig. 柏, b. This is because the coordinate wave function is antisymmetric with respect to the permutation of electrons and hence the electrons are well separated in space.

\section{THE MULTI-ELECTRON CIRCULAR QUANTUM DOT}

The second difference of the total energy is defined in the usual way

$$
\Delta_{2}(N)=E(N+1)+E(N-1)-2 E(N),
$$

where $N$ is the number of electrons in the dot. Fig. 6 shows calculated values of $\Delta_{2}$ against $N$ in the round dot with confinement frequency $\omega_{\mathrm{at}}=0.73$ (atomic units) which corresponds to $r_{s}=1.48 \ldots 0.99$ over the range of $N$ (see Eq. 9). We also find the ground state spin. Values of the spin at even values of $N$ are marked above the lines. 

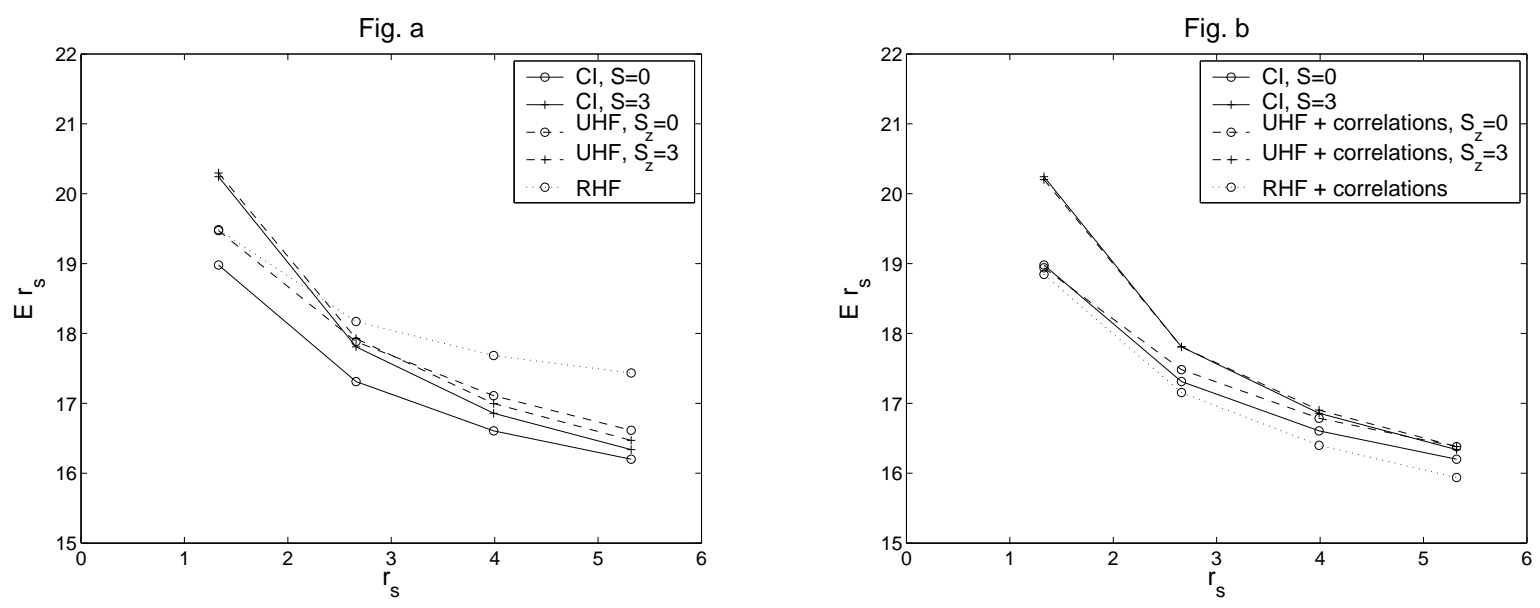

FIG. 5: Total energy versus $r_{s}$ for the six particle problem, with CI energies taken from the configuration-interaction study of Reimann et $a l^{5}$. We plot the total energy in atomic units multiplied by $r_{s}$. Exact solutions for $S=0$ and $S=3$ are shown in both Fig.a and Fig.b by solid lines. Fig.a also shows the UHF energy with $S_{z}=0$ and $S_{z}=3$ (the dashed lines) and the RHF energy (the dotted line). Fig.b shows the same energies with second-order correlation terms (20) included in all cases. Lines joining data points are given as a guide to the eye only.

Fig. a

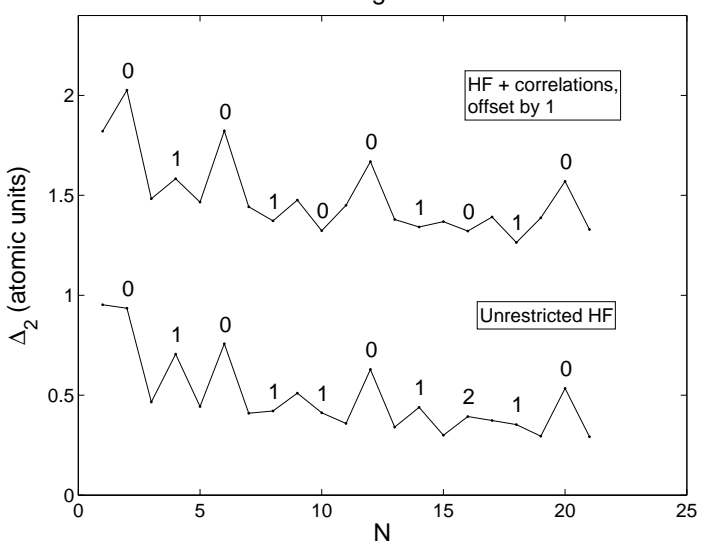

Fig. b

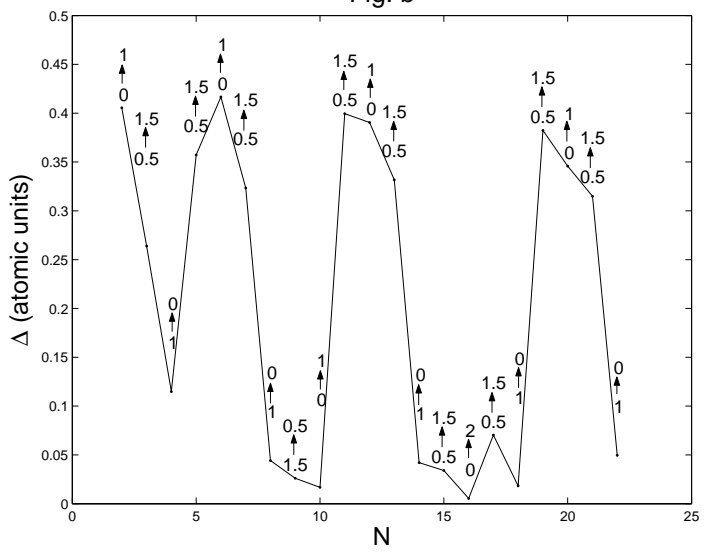

FIG. 6: The circular dot with confinement frequency $\omega_{\text {at }}=0.73$ which corresponds to $r_{s}=1.48 \ldots 0.99$ over the range of $N$. a: $\Delta_{2}$ against $N$ from unrestricted HF (bottom line) and from $\mathrm{HF}+$ correlations (top line). At even values of $N$ we also show the spin of the ground state.

b: The excitation gap with spin change against $N$ (correlations are taken into account). At each $\mathrm{N}$ we also show the spin of the ground state and the spin of the excited state.

The bottom line in Fig. [6 describes naive HF calculations with no correlations included. These are all UHF results since the raw UHF energy will always be lower than the raw RHF energy. To determine total spin of the UHF state we perform calculations for different values of $S_{z}$ and set $S$ to the highest of the $S_{z}$ which give degenerate energies. In cases when $S \neq 0$, we see very degenerate energies up to the maximal spin, followed by a much larger energy gap for $S_{z}>S$, and this indicates that UHF almost preserves the rotational invariance. These UHF results agree with those obtained previously using spin density functional theory ${ }^{4.6}$. The $2 \mathrm{D}$ parabolic shell structure is reflected in the $\Delta_{2}$ values. We see peaks at $N=2,6,12$, and 20 , consistent with the shell model $1 s^{2} 2 p^{4} 3 s^{2} 3 d^{4} 4 p^{4} 4 f^{4}$. As one would expect, the Hartree-Fock spin structure agrees with that predicted by Hund's rule.

The top line (offset) in Fig. 6a shows results obtained by taking correlations into account. At each $N$ we perform $\mathrm{UHF}+$ correlation calculations for different values of $S_{z}$ and we also perform a RHF+correlation calculation for $S=0$ (or $S=\frac{1}{2}$ if $N$ is odd). This allows us to determine the ground state energy and spin. Fig. 6a shows that correlations influence the values of $\Delta_{2}$ for some $N$, but retain the shell structure. For some $N$ where the shell is open, i.e. $N=10$, 
Fig. a

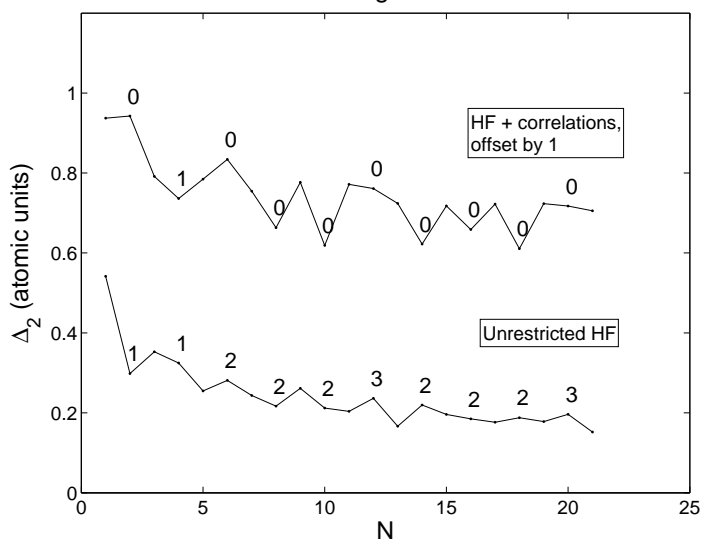

Fig. b

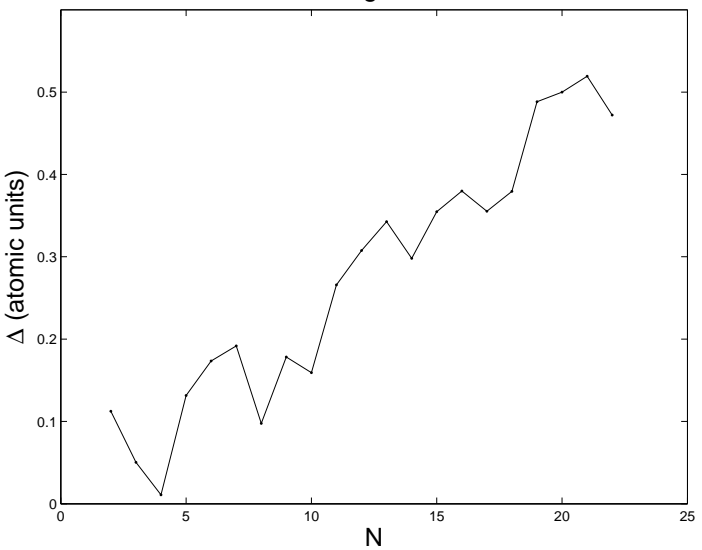

FIG. 7: The circular dot with confinement frequency $\omega_{\text {at }}=0.26$ which corresponds to $r_{s}=2.91 \ldots 1.95$ over the range of $N$. a: $\Delta_{2}$ against $N$ from unrestricted HF (bottom line) and from HF+correlations (top line). At even values of $N$ we also show the spin of the ground state.

b: The excitation gap against $N$ as defined by $E_{\mathrm{UHF}, \mathrm{Sz}=1}-E_{\mathrm{RHF}, \mathrm{S}=0}$ (correlations are taken into account).

$15,16,17$, the correlations also change the spin from $1, \frac{3}{2}, 2, \frac{3}{2}$ to $0, \frac{1}{2}, 0, \frac{1}{2}$. In these cases, including correlations leads to a violation of Hund's rule. However, in these cases we see that the excitation gap with change of spin

$$
\Delta=E_{1}(N)-E_{0}(N)
$$

is very small in these dots. The spin gap becomes large only for $N$ corresponding to closed shells. Values of $\Delta$ versus $N$ at $\omega_{\text {at }}=0.73$, with correlations taken into account, are plotted in Fig. 6b. Unfortunately our technique does not allow the calculation of the gap without spin change. Note that this gap is not equal to the difference of the single particle Hartree-Fock energies. We should point out that at $N=16$, where the calculated excitation goes from $S=0$ to $S=2$, we cannot accurately calculate the energy at $S=1$ as only $S_{z}$ is conserved in the HF method. This means we cannot observe the $S=1$ excitation - most likely it has energy comparable to that of the $S=2$ excitation.

Fig. 7 shows the same quantities $\Delta_{2}$ and $\Delta$ for dots with weaker confinement $\omega_{\text {at }}=0.26$. This corresponds to stronger interaction, $r_{s}=2.91 \ldots 1.95$ over the range of $N$. In this case correlations substantially influence the values of $\Delta_{2}$, although the shell structure on $\Delta_{2}$ is destroyed by electron-electron interactions whether or not correlations are included. It should be noted that in the UHF case here it becomes difficult to determine which energies should be treated as degenerate and which should not. The UHF spin values given on the bottom line of Fig. $7 \mathrm{~h}$ should be taken with caution. This is in contrast to Fig. [6 where the degeneracies are clear-cut. When correlations are included, the ground state spin becomes minimal for almost all $N$. We see in Fig [7b that the "spin gap" of Eq. (28) grows linearly with $N$. At the same time, the UHF+correlations $S_{z}=1$ calculation in this case generates a highly excited state with a strong spin density wave (the spin separation parameter is $\Delta n \sim 4-5$ ). This implies that the accuracy of the UHF+correlations method drops dramatically at the larger $N$ due to the developement of unphysical spin density waves, and that the gap in Fig. 7b is not a true spin gap. We believe that the $S=0$ ground state, as determined by RHF+correlations, is fairly accurate. In principle, based on this ground state, one could find the first physical excitation using the time-dependent $\mathrm{HF}+$ correlations method. However this is a very involved calculation and beyond the scope of the present work.

\section{ELLIPTICAL DOTS}

Studies of elliptical dots are interesting from a theoretical point of view. Also, an elliptical deformation is a first way to model deviations from circular geometry, which always exist in real dots. A lateral dot, for instance, has contacts at the sides through which the electrons tunnel onto the dot, and these alter the shape of the confining potential. Previous numerical studies have found that a deformation of the dot destroys the shell structure $\stackrel{7.8,10}{=}$ In the present work we investigate a simple deformation of the dot by taking $\omega_{x}>\omega_{y}$.

Fig. 8 a shows calculated values of $\Delta_{2}$ against $N$ for a dot with the small ellipticity $\omega_{x} / \omega_{y}=1.1$ and with $\omega_{\text {at }}=\sqrt{\omega_{x} \omega_{y}}=0.73$, which corresponds to $r_{s}=1.48 \ldots 0.99$ over the range of $N$. The value of the ground state spin 
Fig. a

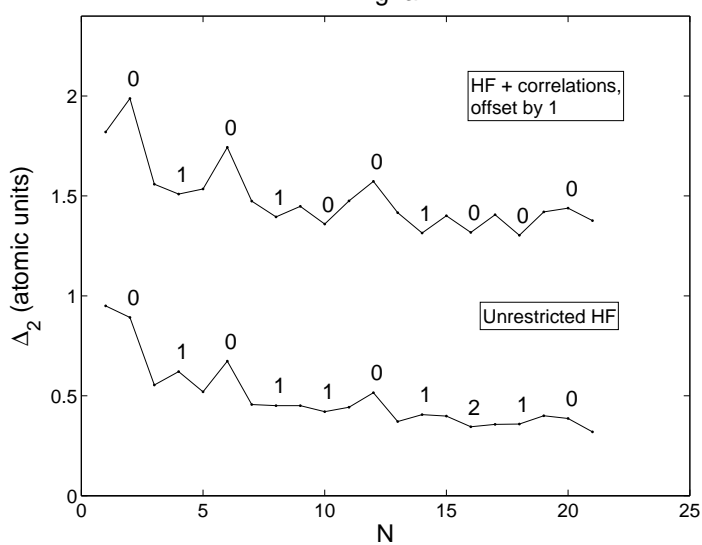

Fig. b

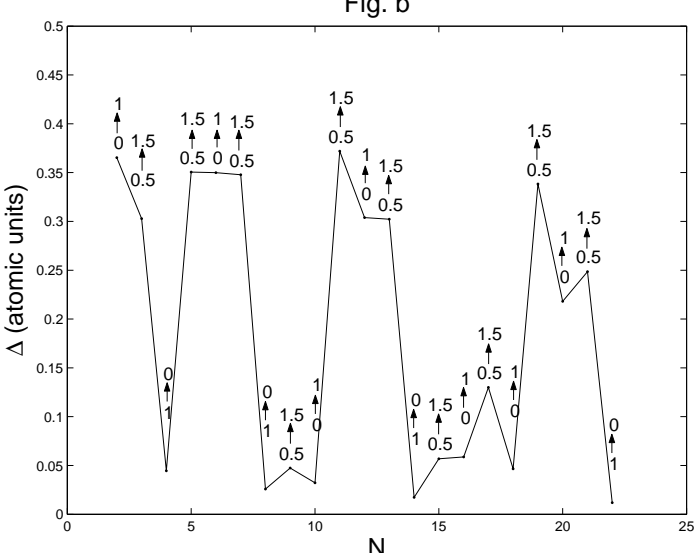

FIG. 8: The dot with ellipticity $\omega_{x} / \omega_{y}=1.1$ and with $\omega_{\text {at }}=\sqrt{\omega_{x} \omega_{y}}=0.73$ which corresponds to $r_{s}=1.48 \ldots 0.99$.

a: $\Delta_{2}$ against $N$ from unrestricted HF (bottom line) and from HF+correlations (top line). At even values of $N$ we also show the spin of the ground state.

b: The excitation gap with spin change against $N$ (correlations are taken into account). At each $\mathrm{N}$ we also show the spin of the ground state and the spin of the excited state.

Fig. a

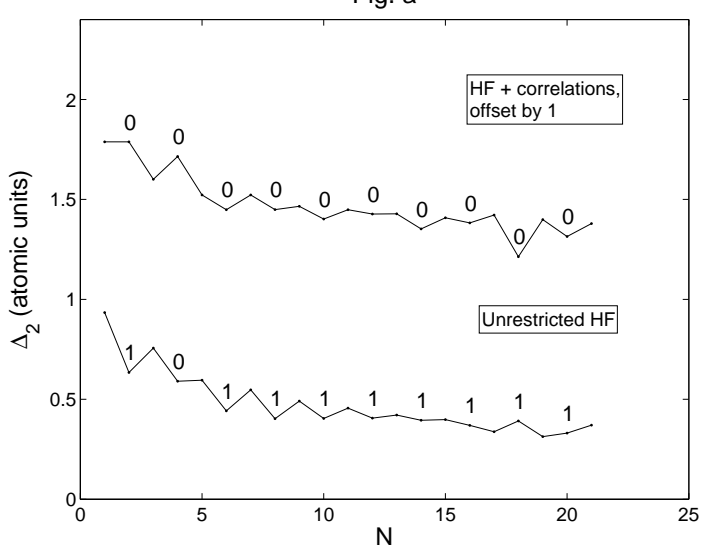

Fig. b

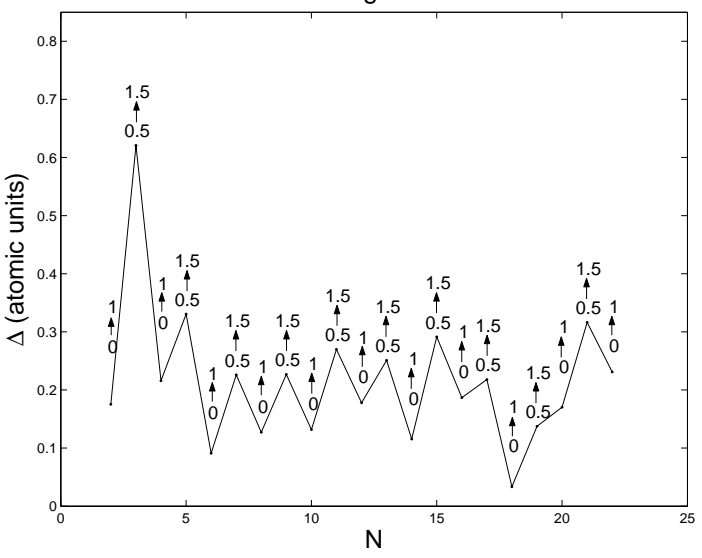

FIG. 9: The dot with ellipticity $\omega_{x} / \omega_{y}=2$ and with $\omega_{\text {at }}=\sqrt{\omega_{x} \omega_{y}}=0.73$ which corresponds to $r_{s}=1.48 \ldots 0.99$.

a: $\Delta_{2}$ against $N$ from unrestricted $\mathrm{HF}$ (bottom line) and from HF+correlations (top line). At even values of $N$ we also show the spin of the ground state.

b: The excitation gap with spin change against $N$ (correlations are taken into account). At each $\mathrm{N}$ we also show the spin of the ground state and the spin of the excited state.

at even values of $N$ is marked above the lines. The bottom line in Fig. 8 8 shows the results of UHF calculations without correlations, and the top line (offset) in Fig. 8 presents values of $\Delta_{2}$ obtained by taking correlations into account. Fig. 8b presents the excitation gap with correlations included. As in the round dot, including correlation effects reduces the ground state spin in several cases and hence leads to the violation of Hund's rule. In this case the excitation gaps for these $N$ can be larger, yet the ground state spin still changes to the minimal value. However, most of the $\Delta_{2}$ peaks given by the shell structure are still visible. The UHF results of Fig. 80 are qualitatively similar, although not identical, to the mean-field (density functional theory) results of Austing et $a l^{\frac{7}{7}}$ for the $\omega_{x} / \omega_{y}=1.1$ dot.

Fig. 9] shows the same quantities $\Delta_{2}$ and $\Delta$ for for dot with large ellipticity $\omega_{x} / \omega_{y}=2$ and with $\omega_{\text {at }}=\sqrt{\omega_{x} \omega_{y}}=0.73$. The range for $r_{s}$ over $N$ remains the same, $r_{s}=1.48 \ldots 0.99$. As in Fig. 7 , the spin identification within UHF is ambiguous and should be treated with caution. The ambiguity disappears when correlations are included. It is known that there are shells in a parabolic potential with $\omega_{x} / \omega_{y}=2$ (see Ref ${ }^{16}$ ). However the self-consistent potential is 
not parabolic and we do not observe a shell structure in the dot, with or without correlations. This is evident from the gap values plotted in Fig. 9 p. However, there is some peculiarity at $N=17,18$, and 19 and this may perhaps be considered as reminiscent of the shell structure. The $\omega_{x} / \omega_{y}=2$ case was also included in the mean-field study of Austing et al $\stackrel{7}{n}$ and no clear signature of the shell structure was observed.

\section{CONCLUSIONS}

We have performed calculations for small quantum dots $(N \leq 22)$ taking into account electron-electron correlations in the second order of perturbation theory. We believe that this approach is well justified for $r_{s} \leq 3$ when the correlation correction does not exceed a few percent of the total energy. To check the method we have performed a comparison with accurate solutions for two and six electrons, in which the method was valid to beyond $r_{s}=3$. We demonstrate that correlations are especially important for the spin structure of the dot. In some cases even at $r_{s}=1$ correlations change the spin of the ground state from that found in Hartree-Fock and spin-density-functional calculations. In some situations correlations destroy Hund's rule for open electronic shells. Finally, we observe that correlations destroy the static spin-density waves observed in Hartree-Fock and spin-density-functional calculations and hence restore the spin rotational invariance of states with total spin zero.

We thank P. G. Silvestrov and A. Micolich for helpful discussions. We are also grateful for the computing resources provided by the Australian Partnership for Advanced Computing (APAC) National Facility.

1 S. Tarucha, D. G. Austing, T. Honda, R. J. van der Hage, and L. P. Kouwenhoven, Phys. Rev. Lett. 77, 3613 (1996).

${ }^{2}$ L. P. Kouwenhoven, T. H. Oosterkamp, M. W. S. Danoesastro, M. Eto, D. G. Austing, T. Honda, and S. Tarucha, Science 278, 1788 (1997).

3 Y. Alhassid, Rev. Mod. Phys. 72, 895 (2000).

${ }^{4}$ S. M. Reimann and M. Manninen, Rev. Mod. Phys. 74, 1283 (2002).

5 S. M. Reimann, M. Koskinen, and M. Manninen, Phys. Rev. B 62, 8108 (2000).

6 M. Koskinen, M. Manninen, and S. M. Reimann, Phys. Rev. Lett. 79, 1389 (1997)

7 D. G. Austing, S. Sasaki, S. Tarucha, S. M. Reimann, M. Koskinen, and M. Manninen, Phys. Rev. B 60, 11514 (1999)

8 In-Ho Lee, Vivek Rao, Richard M. Martin, and Jean-Pierre Leburton, Phys. Rev. B. 57, 9035 (1998).

9 S. M. Reimann, M. Koskinen, J. Helgesson, P. E. Lindelof, and M. Manninen, Phys. Rev. B 58, 8111 (1998).

${ }^{10}$ K. Hirose and N. S. Wingreen, Phys. Rev. B 59, 4604 (1999).

11 T. F. Jiang, Xiao-Min Tong, and Shih-I Chu, Phys. Rev. B. 63, 045317 (2001).

12 I. I. Yakimenko, A. M. Bychkov, and K. -F. Berggren, Phys. Rev. B 63, 165309. (2001).

13 C. Yannouleas and U. Landman, Phys. Rev. Lett. 82, 5325 (1999).

14 C. Yannouleas and U. Landman, J. Phys.: Condens. Matter 14, L591 (2002)

15 A. W. Overhauser, Phys. Rev. 128, 1437 (1962).

16 A. Bohr and B. Mottelson, Nuclear Structure vol. 2.

17 I. A. Sneddon, Mixed Boundary Value Problems in Potential Theory (Wiley, New York, 1966).

18 A. A. Koulakov and B. I. Shklovskii, Phys. Rev. B 57, 2352 (1998).

19 E. Cancès and C. Le Bris, Int. J. Quantum Chem. 79, 82-90 (2000).

20 M. Gell-Mann and K. A. Brueckner, Phys. Rev. 106, 364 (1957).

21 Strictly speaking at $N=2, r_{s}$ is not a well defined quantity since Eq. (9) was derived at $N \gg 1$. Nevertheless it is convenient to use $r_{s}$ defined according to (9) as an appropriate scaling variable.

${ }^{22}$ V. A. Dzuba, V. V. Flambaum, and O. P. Sushkov, Phys. Lett. A 141, 147-153 (1989).

23 S. A. Blundell, W. R. Johnson, and J. Sapirstein, Phys. Rev. Lett. 65, 1411 (1990).

24 W. R. Johnson, H. C. Ho, C. E. Tanner, and A. Derevianko, Phys. Rev. A 70, 014501 (2004). 